\title{
New Zealand dietary patterns in relation to bone and nutritional health status among post-menopausal women
}

\author{
$\underline{B o l a j i}$ Lilian Ilesanmi-Oyelere $^{1,2}$, Louise Brough ${ }^{1}$, Jane Coad $^{1}$, Nicole $\operatorname{Roy}^{2,3}$ and \\ Marlena Kruger ${ }^{1,3}$ \\ ${ }^{1}$ Massey University, Palmerston North, New Zealand., \\ ${ }^{2}$ AgResearch, Grasslands, Palmerston North, New Zealand. and \\ ${ }^{3}$ Riddet Institute, Palmerston North, New Zealand
}

\section{Abstract}

Nutrients affect bone health status. However, dietary patterns advance insight into which particular foods influence bone and nutritional health status. The aim of this study was to explore the associations between dietary patterns, bone and nutritional health status in postmenopausal women. This cross-sectional study examined 127 postmenopausal women aged between 54-81 years. Body composition and bone health status were determined using dual-energy X-ray absorptiometry (DXA). The dietary composition was assessed by a validated food frequency questionnaire composed of 108 foods, of which 34 food groups were created. Dietary patterns (DP) were identified by principal component analysis. The bone and nutritional measures were regressed onto the dietary pattern. Five DPs were identified, two of which were associated with nutritional status. Fruit and carbohydrate DP was associated with decreased weight $(\mathrm{r}=-0.192, P=0.038)$, body mass index $(\mathrm{r}=-0.184, P=0.047)$ and body fat $(\mathrm{r}=-0.181, P=0.050)$. Coffee and yoghurt $/ \mathrm{cream}$ DP was associated with weight $(\mathrm{r}=0.223, P=0.015)$, body mass index $(\mathrm{r}=0.245, P=0.008)$ and body fat $(\mathrm{r}=0.195, P=0.036)$. Bone health status; spine T-score $(\mathrm{r}=0.283, P=0.002)$, hip T-score $(\mathrm{r}=0.309, P=0.001)$, femoral neck $\mathrm{T}$-score $(\mathrm{r}=0.317, P=0.001)$ and whole-body bone mineral density $(\mathrm{r}=0.261, P=0.004)$ were also associated with coffee and yoghurt/cream DP. Milk products DP was associated with spine $\mathrm{T}$-score $(\mathrm{r}=0.304, P=0.001)$. A dietary pattern characterized by high factor loadings of coffee, milk and yoghurt/cream was associated with higher BMD and T-scores. Although coffee intake has been associated with low bone mass, the impact of milk products appears to override the effects of coffee as an important determinant of bone health status in postmenopausal women.

\section{Conflict of Interest}

There is no conflict of interest. 